\title{
Control of speed and efficiency of ultrafast demagnetization by direct transfer of spin angular momentum
}

\author{
G. MALINOWSKI*, F. DALLA LONGA, J. H. H. RIETJENS, P. V. PALUSKAR, R. HUIJINK, H. J. M. SWAGTEN \\ AND B. KOOPMANS
}

Department of Applied Physics, Center for NanoMaterials, Eindhoven University of Technology, PO Box 513, 5600 MB Eindhoven, The Netherlands

*e-mail: g.malinowski@tue.nl

Since the discovery in 1996 that the magnetization of a nickel thin film is reduced within a few picoseconds after femtosecond-laser excitation ${ }^{1}$, ultrafast demagnetization has attracted a thriving interest. This attraction is driven by the twofold challenge of understanding magnetization dynamics in a strongly out-of-equilibrium regime and controlling the magnetic properties of materials on the subpicosecond timescale, with potential applications in spintronics. In the past decade significant progress has been made in understanding the microscopic processes that govern ultrafast demagnetization ${ }^{2-7}$. The discussion has been particularly focused on the role of angular-momentum conservation during the demagnetization process $^{8-10}$. Here, using the time-resolved magneto-optical Kerr effect, we demonstrate that interlayer transfer of spin angular momentum in specially engineered $\mathrm{Co} / \mathrm{Pt}$ multilayers speeds up the demagnetization process, bypassing the mechanism responsible for the conservation of total angular momentum taking place in a single ferromagnetic layer. This new channel for spin-angular-momentum dissipation leads to a reduction of the demagnetization time of up to $25 \%$, accompanied by an increase of the total demagnetization by almost the same amount.

Recently, spin torque transfer has been heavily used in spintronics devices to excite or reverse the magnetization of a ferromagnet (for a review see ref. 11) or to move a magnetic domain wall within a ferromagnetic nanowire ${ }^{12,13}$. In these experiments, the torque is created by a spin-polarized charge current. On the other hand, theoretical predictions ${ }^{14,15}$ and experimental proofs ${ }^{14,16}$ have shown that a pure spin current can be created by magnetization dynamics in the absence of a charge current. This mechanism, known as spin pumping, appears at the interface between a ferromagnet with a precessing magnetization and a normal metal, leading to polarization of the electrons and a spin accumulation in the normal metal. Spin pumping was first brought to light as a broadening of the ferromagnetic resonance line due to an increase of the effective Gilbert damping parameter ${ }^{17,18}$. Only recently, Woltersdorf et al. ${ }^{19}$ have shown that the exchange of pure spin current in a ferromagnet-normal metal-ferromagnet leads to magnetization dynamics. These two effects might be the source of new paths for spin-angular-momentum dissipation and therefore influence the magnetization dynamics of a ferromagnetic metal after pulsed laser excitation. a
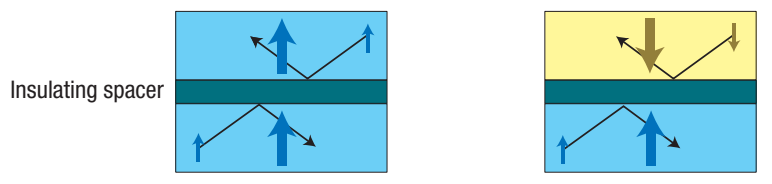

b
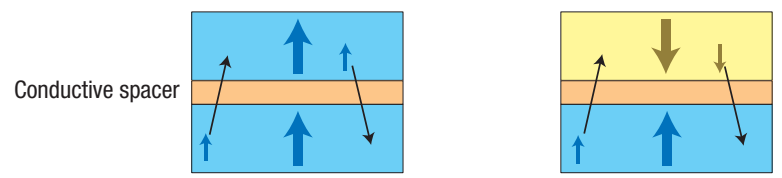

G
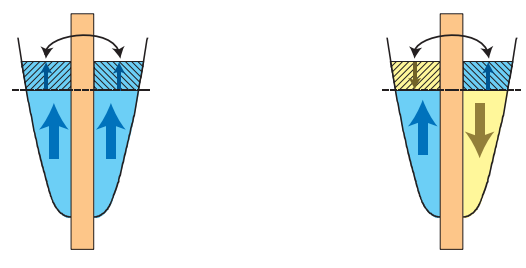

$\delta M_{\text {he }}=0$

$\delta M_{\text {he }}>0$

Figure 1 Schematic diagrams of hot-electron localization and spin-momentum transfer after laser excitation. a, Diagram for an insulating spacer. $\mathbf{b}$, Diagram for a metallic spacer. c, Schematic parabolic band structure of both Co-Pt multilayers. For the sake of clarity, only the majority band is shown. $\delta M_{\text {ne }}$ represents the amount of demagnetization due to hot-electron spin-angular-momentum transfer.

Spin-angular-momentum transfer in ferromagnetic multilayers relies on spin conservation during electronic transport from one ferromagnet to another. Hence, it is possible to control the efficiency of the spin-angular-momentum transfer by inserting a spacer between the two ferromagnetic layers that is either transparent or acts as a barrier for spin-polarized carriers. Let us consider a configuration of two identical ferromagnetic layers separated by a thin layer as described in Fig. 1. After excitation of the system by a laser pulse, hot electrons are created in both ferromagnetic layers. If the spacer acts as a barrier for spin transport, either insulating or with a very short spin scattering 
a

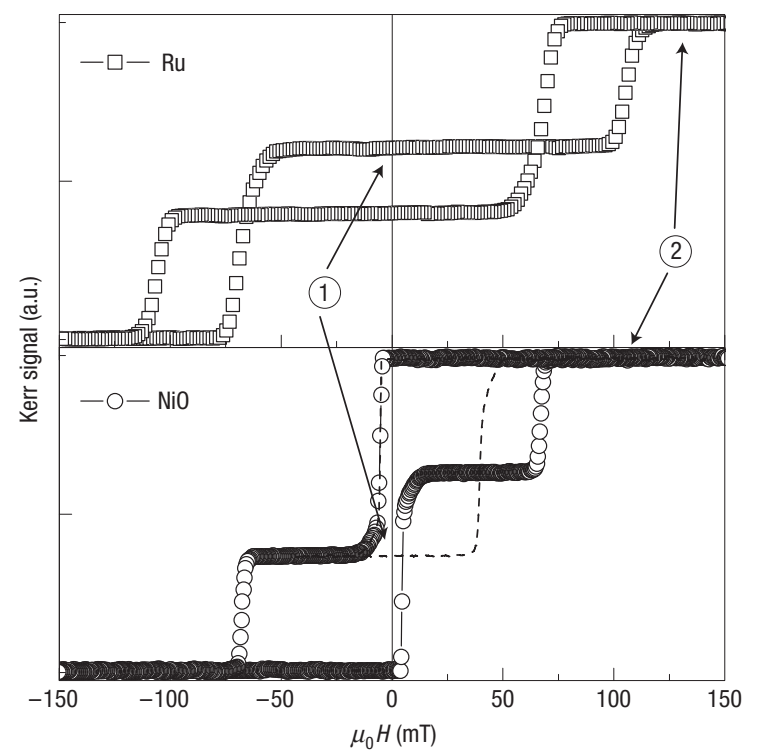

b

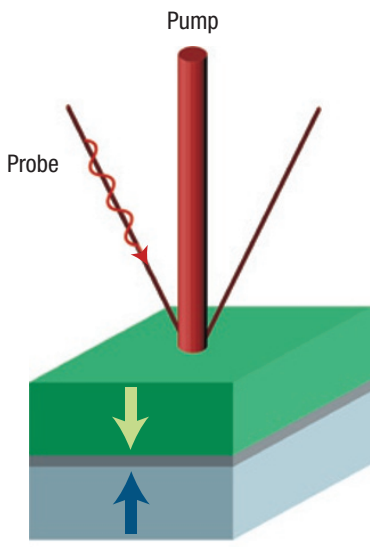

(1) $\mu_{0} H=0$

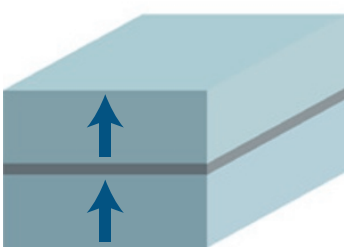

Figure 2 Static magnetic properties and geometry of the samples. a, Hysteresis loops measured by static MOKE on samples with a spacer of $0.4 \mathrm{~nm}$ of Ru and $1.2 \mathrm{~nm}$ of $\mathrm{NiO}$. b, Schematic representation of the experiment. Configurations 1 and 2 respectively represent the antiparallel (AP) and parallel (P) states.

length, independent of the relative orientation of the magnetization of the two layers, no spin current is carried from one layer to the other (Fig. 1a). On the other hand, if the spacer does not provide a barrier, a spin current can flow from the top layer to the bottom one and vice versa (Fig. 1b). In this case, the net spin current depends on the relative orientation of the magnetization of the two ferromagnetic layers. For a parallel (P) alignment, spin currents cancel, resulting in a zero net spin current. In contrast, for an antiparallel (AP) alignment, a net spin current is induced that can efficiently change the ratio between majority and minority spins in each layer. Thereby, opening this additional channel for spin-angular-momentum transfer should lead to a decrease of the net magnetization of both magnetic layers on the femtosecond timescale.

To experimentally explore demagnetization by laser-induced interlayer spin transfer, we have studied specifically chosen identical $[\mathrm{Co}-\mathrm{Pt}]_{n}$ multilayers antiferromagnetically coupled through a thin spacer. This is considered as an ideal system to test this spintransfer mechanism. To start with, because of their perpendicular anisotropy and strong spin-orbit coupling, $[\mathrm{Co}-\mathrm{Pt}]_{n}$ multilayers give rise to a large magneto-optic Kerr effect. Moreover, using two identical ferromagnetic layers simplifies interpretation of the time-resolved magneto-optical Kerr effect (TR-MOKE) results. Stabilization of a $\mathrm{P}$ or AP magnetization configuration requires independent switching of both multilayers which can be obtained by making use of antiferromagnetic coupling. Such a coupling is obtained by separating two $\mathrm{Co}-\mathrm{Pt}$ multilayers by a thin $\mathrm{Ru}$ (refs 20,21) or $\mathrm{NiO}$ spacer $^{22,23}$ (see Supplementary Information, Part I). The use of $\mathrm{Ru}$ and $\mathrm{NiO}$ as different spacers provides the necessary contrasting behaviour regarding spin conservation, because $\mathrm{Ru}$ is a metallic spacer thin enough to enable conservation of spin whereas $\mathrm{NiO}$ is an antiferromagnetic insulating material. Not only do we expect that $\mathrm{NiO}$ will have a high efficiency of blocking the transfer of polarized hot carriers, unlike traditional non-magnetic barriers, but also we explicitly verified its insulating
Table 1 Demagnetization magnitude measured from the demagnetization curves, and demagnetization time $\tau_{M}$ and electron-phonon relaxation time $\tau_{E}$ obtained by averaging the results of the fit of separate demagnetization curves (three to five). The error bars are the standard deviations.

\begin{tabular}{|c|c|c|c|c|c|c|c|c|}
\hline & \multicolumn{3}{|c|}{$\Delta \theta / \theta(\%)$} & \multicolumn{2}{|c|}{$\tau_{E}(\mathrm{fS})$} & \multicolumn{2}{|c|}{$\tau_{M}(\mathrm{fS})$} \\
\hline & & $\mathrm{P}$ & AP & AP (theory) & $P$ & AP & $P$ & AP \\
\hline \multirow{2}{*}{$\mathrm{NiO}$} & $1.2 \mathrm{~nm}$ & -2.0 & -4.0 & -3.8 & & \pm 6 & $78 \pm 2$ & $74 \pm 2$ \\
\hline & $1.7 \mathrm{~nm}$ & -2.0 & -3.9 & -3.8 & & \pm 6 & $74 \pm 2$ & $72 \pm 2$ \\
\hline \multirow{2}{*}{$\mathrm{Ru}$} & $0.4 \mathrm{~nm}$ & -2.2 & -5.2 & -4.2 & & \pm 4 & $95 \pm 2$ & $71 \pm 2$ \\
\hline & $0.9 \mathrm{~nm}$ & -2.2 & -4.9 & -4.2 & & \pm 4 & $95 \pm 2$ & $79 \pm 2$ \\
\hline
\end{tabular}

behaviour when embedded in our multilayered structure (see Supplementary information, Part II).

Static MOKE hysteresis loops measured on samples with $0.4 \mathrm{~nm}$ of $\mathrm{Ru}$ and $1.2 \mathrm{~nm}$ of $\mathrm{NiO}$ are shown in Fig. 2a (see the Methods section). The two Co-Pt multilayers are antiferromagnetically coupled and show two distinct switching fields separated by a large antiferromagnetic plateau for both $\mathrm{Ru}$ and $\mathrm{NiO}$ spacers. The magnetization directions for both $\mathrm{Co}-\mathrm{Pt}$ multilayers are depicted in Fig. 2b for different magnetic applied fields. When the applied magnetic field is large enough to overcome the antiferromagnetic coupling, the magnetizations of both multilayers are aligned in the same direction ( $\mathrm{P}$ state). However, when the applied magnetic field is smaller than the interlayer exchange coupling, their magnetization directions are antiparallel (AP state). The artificial antiferromagnets are fully compensated because of the two identical Co-Pt multilayers used in every sample. Thereby, the net magnetization in the AP state is null. However, a non-zero MOKE signal is observed owing to different Kerr sensitivities of each layer as a result of the absorption profile in the multilayer. 

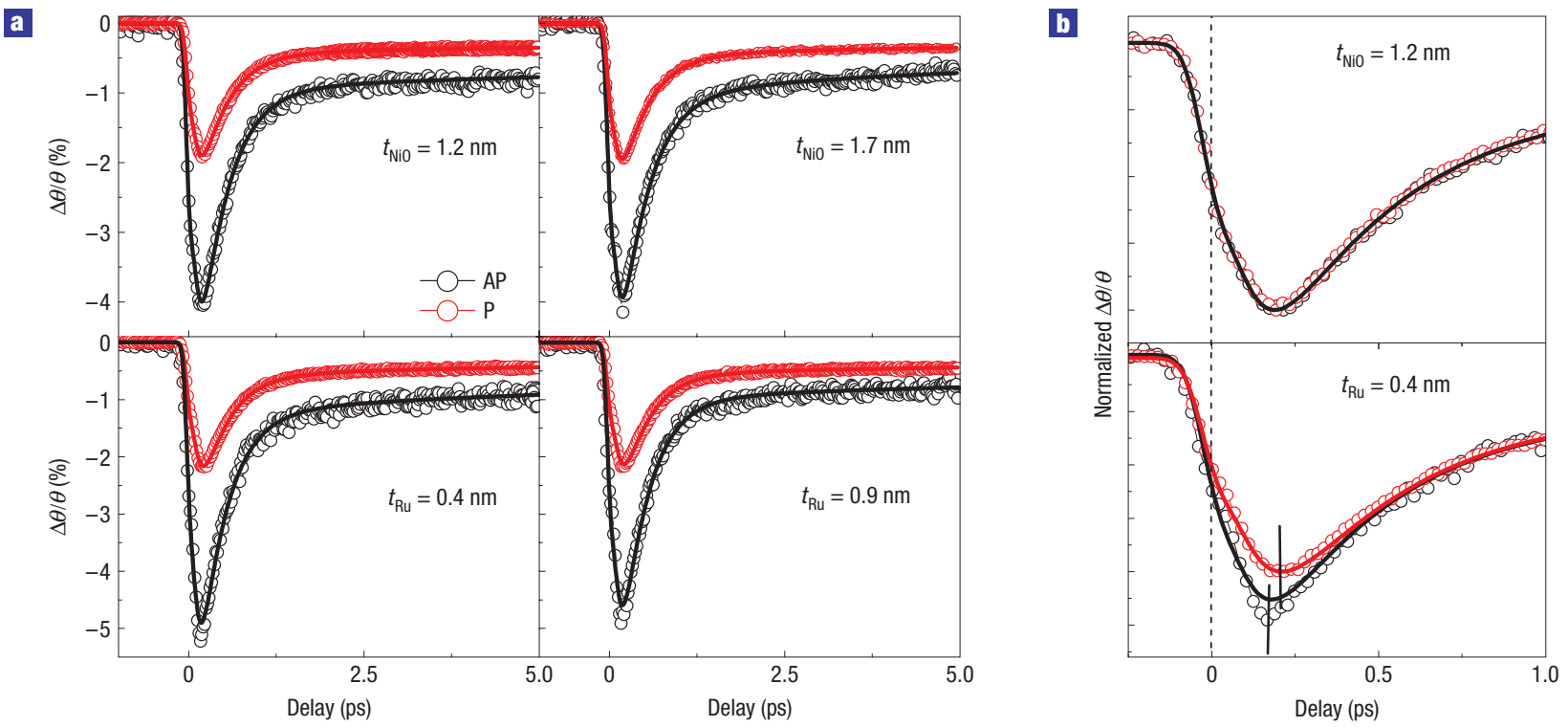

Figure 3 TR-MOKE measurements. a, TR-MOKE measured in the parallel and antiparallel states for different thicknesses of NiO (1.2 $\mathrm{nm}$ and $1.7 \mathrm{~nm})$ and Ru (0.4 nm and $0.9 \mathrm{~nm}$ ). b, Normalized TR-MOKE measurement for $1.2 \mathrm{~nm}$ of NiO and $0.4 \mathrm{~nm}$ of Ru. Curves are normalized at $3 \mathrm{ps}$, after electron thermalization. The solid lines are fits to the data (see the Methods section).

The top Co-Pt multilayer is responsible for $60 \%$ of the total Kerr signal. We calculated the absorption profile of our samples and we found that the ratio between the absorbed light in the top and the bottom multilayers is for each spacer close to 1.5 (see Supplementary Information, Part III). Thus, the ratio of the MOKE signal corresponding to the reversal of the magnetization of both multilayers is similar to the ratio of absorbed light within these layers.

Typical TR-MOKE measurements showing the pump-induced change of the Kerr rotation are depicted in Fig. $3 \mathrm{a}$ for two $\mathrm{Ru}$ and $\mathrm{NiO}$ thicknesses. We used a relatively low laser fluence of

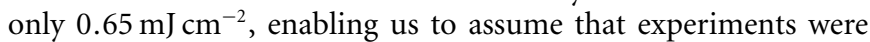
performed in the linear regime, where the change in the Kerr rotation is directly proportional to the change in the perpendicular magnetic moment. For every spacer thickness, measurements were carried out in both $\mathrm{P}$ and AP states. Interestingly, a striking difference between normalized curves measured in the $\mathrm{P}$ and AP states is seen only when a Ru spacer is used (Fig. 3b). In this case, a larger magnetization loss combined with a faster demagnetization is observed in the AP state. In general, magnetization dynamics can be described using the so-called three-temperature model ${ }^{1,7,10}$ (see the Methods section). This phenomenological model pictures how energy is redistributed among electrons, spins and the lattice after the laser power has been absorbed by the electronic system. The heat flow ultimately leads to an increase of the spin temperature, and thereby reduces the magnetization. Fitting our measurements using this model enables us to extract the characteristic demagnetization time $\tau_{M}$ and the electron-phonon relaxation time $\tau_{E}$ (Table 1 ). We would like to stress that the parameter $\tau_{M}$ incorporates, in an effective way, different mechanisms responsible for the loss of magnetization, without the need of previous knowledge of which is dominant ${ }^{7}$. The averaged values for the electron-phonon relaxation time are $\bar{\tau}_{E}^{\mathrm{NiO}}=396 \pm 6 \mathrm{fs}$ and $\bar{\tau}_{E}^{\mathrm{Ru}}=372 \pm 4 \mathrm{fs}$ respectively for $\mathrm{NiO}$ and $\mathrm{Ru}$, and they are identical for both magnetic configurations. These values are in good agreement with previous measurements on other ferromagnetic transition metals ${ }^{24}$. More interestingly, the demagnetization time
$\tau_{M}$ depends on both the spacer and the magnetic configuration. For both thicknesses of $\mathrm{NiO}$, similar $\tau_{M}$ values were extracted from the fits with an average value of $\bar{\tau}_{M}^{\mathrm{NiO}}=75 \pm 2 \mathrm{fs}$ independent of the magnetic state. In contrast, when a $\mathrm{Ru}$ layer is used, a difference in the demagnetization time is seen between the $\mathrm{P}$ and AP states (Fig. 3b). When both magnetization directions are parallel, a larger demagnetization time of $\tau_{M}^{\mathrm{Ru}}(\mathrm{P})=95 \pm 2 \mathrm{fs}$ is measured in comparison with $\tau_{M}^{\mathrm{Ru}}(\mathrm{AP})=71 \pm 2 \mathrm{fs}$ and $\tau_{M}^{\mathrm{Ru}}(\mathrm{AP})=79 \pm 2 \mathrm{fs}$ in the AP configuration for $0.4 \mathrm{~nm}$ and $0.9 \mathrm{~nm}$ of Ru respectively. This decrease by $25 \%$ of the demagnetization time in the AP state can be ascribed to direct spin momentum transfer between the two Co-Pt layers. The small difference in $\tau_{M}$ and $\tau_{E}$ observed between samples with either a $\mathrm{Ru}$ or a $\mathrm{NiO}$ spacer might be related to a change in hot-electron transport and heat diffusion between the two Co-Pt multilayers ${ }^{24}$ and effects such as interface-dependent spin-flip scattering, but it is not of relevance in the present work.

In addition to a faster demagnetization, transfer of spin angular momentum could also influence the amount of magnetization loss. To assess this point, we compare the relative change in the Kerr rotation $\Delta \theta / \theta$ at the peak position around $0.2 \mathrm{ps}$. In the parallel state, a similar change of $(\Delta \theta / \theta)_{\mathrm{P}} \simeq-2.0 \%$ is observed irrespective of the spacer and its thickness. However, this is no longer the case in the AP state. First, a larger change in the Kerr rotation is measured for both spacers. As mentioned earlier, this increase is due to the depth dependence of the Kerr signal because of the absorption profile of the laser in the sample. Second, compared with $\mathrm{NiO}$, a larger change of $(\Delta \theta / \theta)_{\mathrm{AP}}$ is seen for both $\mathrm{Ru}$ thicknesses and is clearly visible after normalization of the TRMOKE curves (Fig. 3b). Taking into account the absorption profile, it is possible to estimate $(\Delta \theta / \theta)_{\mathrm{AP}}$ using the value of $(\Delta \theta / \theta)_{\mathrm{P}}$ (see the Methods section). In the case of $\mathrm{NiO}$, we observe a very good agreement between measurements and estimates $(0.2 \%)$. In contrast, for $\mathrm{Ru}$, a large discrepancy appears with a difference between measurements and estimates, $(\Delta \theta / \theta)_{\mathrm{AP}}^{\exp }-(\Delta \theta / \theta)_{\mathrm{AP}}^{\text {theory }}$, of $-1 \%$ and $-0.7 \%$ for $0.4 \mathrm{~nm}$ and $0.9 \mathrm{~nm}$ respectively. This increase in the amount of demagnetization of $(5.2-4.2) / 4.2 \sim 25 \%$ in the AP state is in line with the decrease of the demagnetization 
time. These results clearly demonstrate that the demagnetization time as well as the amount of demagnetization can be tuned in a ferromagnetic layer by controlling the transfer of spin angular momentum. We emphasize that after equilibration $(\sim 0.5 \mathrm{ps})$ the enhanced demagnetization due to the transfer of spin vanishes, and as expected thermalized $\mathrm{AP}$ and $\mathrm{P}$ curves converge.

Finally, we would like to draw attention to the difference in the amount of demagnetization and demagnetization time observed in the AP state for both Ru thicknesses. We noticed a reduction in the amplitude of the demagnetization accompanied by an enhancement of the demagnetization time when the $\mathrm{Ru}$ thickness was increased from 0.4 to $0.9 \mathrm{~nm}$. This decrease could be attributed to a less efficient transfer of the spin angular momentum from one layer to another when the Ru thickness is increased owing to the short spin diffusion length of $\mathrm{Ru}(4 \mathrm{~nm}$ at $300 \mathrm{~K}$ ) (ref. 25). Although this change is clearly measurable and reproducible, care has to be taken before drawing a final conclusion and more experiments are needed to corroborate or deny this hypothesis.

\section{METHODS}

\section{SAMPLE DESCRIPTION AND PUMP-PROBE EXPERIMENT}

Samples are grown by d.c. magnetron sputtering onto Si substrates with a native oxide. Stacks consist of $10 \mathrm{Pt}-[0.4 \mathrm{Co}-0.7 \mathrm{Pt}]_{4}-0.4 \mathrm{Co}-t \mathrm{~S}-0.4 \mathrm{Co} /[0.7$ $\mathrm{Pt}-0.4 \mathrm{Co}]_{4}-2 \mathrm{Pt}$ (units in nanometres), with $\mathrm{S}$ being either $\mathrm{Ru}$ or $\mathrm{NiO}$ of thickness $t$. Static MOKE experiments were carried out using a HeNe laser with a wavelength of $632.8 \mathrm{~nm}$. The electrical properties of our $\mathrm{NiO}$ were verified using the technique of current-in-plane tunnelling. These measurements were carried out on a microprobe tool, using an array of 12 microscopic probes from CAPRES, specifically developed for this technique ${ }^{26}$ (see Supplementary Information I and II). TR-MOKE experiments are realized using a pump-probe technique. Pump and probe are issued from the same laser. We use a beam splitter to split the laser into a strong (pump) and a weak (probe) pulse of $70 \mathrm{fs}$. The two beams hit the sample at almost normal incidence $\left(\sim 3^{\circ}\right.$ from the normal) and they are focused down to the same $8 \mu \mathrm{m}$ spot. In this geometry, the Kerr rotation is sensitive only to the variation in the out-of-plane component of the magnetization. The laser wavelength is $785 \mathrm{~nm}$, corresponding to an energy of $1.6 \mathrm{eV}$. Time-resolved measurements are made by adjusting the delay between the pump and the probe lasers with a minimum step size of $2 \mathrm{fs}$. In the $\mathrm{P}$ state, samples are saturated under a magnetic field of $\pm 150 \mathrm{mT}$. In the AP state, first we take care to reverse the top Co-Pt magnetization before switching the applied field to zero.

\section{THREE-TEMPERATURE MODEL}

The dynamics of the spin fluctuations can be described by a phenomenological thermodynamic model, the so-called three-temperature model ${ }^{1,7,10}$. This model is based on the separation of the system into three subsystems: the electron, lattice and spin systems. On laser excitation, hot electrons are instantaneously created. After thermalization by electron-electron interactions, each system is internally in thermal equilibrium and it can be described by its own thermodynamic temperature. The relaxation takes place by balancing the different subsystems' temperatures, leading to a flow of energy between them. The set of differential equations describing the heat flow between the three subsystems is solved ignoring the spin specific heat, assuming that the measurements are done in the low-laser-fluence limit and that the electron temperature rises instantaneously on laser excitation ${ }^{7}$ :

$$
\begin{aligned}
-\frac{\Delta M_{z}}{M_{z}}= & {\left[\left(\frac{A_{1}}{\left(t / \tau_{0}+1\right)^{1 / 2}}-\frac{\left(A_{2} \tau_{E}-A_{1} \tau_{M}\right) \mathrm{e}^{-\frac{t}{\tau_{M}}}}{\tau_{E}-\tau_{M}}\right.\right.} \\
& \left.\left.-\frac{\tau_{E}\left(A_{1}-A_{2}\right) \mathrm{e}^{-\frac{t}{\tau_{E}}}}{\tau_{E}-\tau_{M}}\right) \Theta(t)+A_{3} \delta(t)\right] \otimes \Gamma(t),
\end{aligned}
$$

where $\Gamma(t)$ is the Gaussian laser pulse, $\Theta(t)$ is the step function and $\delta(t)$ is the Dirac delta function. The constant $A_{1}$ represents the value of $-\Delta M_{z} / M_{z}$ after equilibrium between electrons, spins and lattice is restored. The constant $A_{2}$ is proportional to the initial electron temperature rise. The constant $A_{3}$ represents the magnitude of state-filling effects during pump-probe temporal overlap, which can be well described by a delta function. Although this model does not take into account conservation of angular momentum, it can prove useful in analysing experimental data and extracting quantitative information on the timescales of the different processes taking place during the relaxation.

\section{ESTIMATION OF THE KERR ROTATION VARIATION AFTER LASER PULSE EXCITATION}

By analysing static MOKE hysteresis loops shown in Fig. 1a, we can infer that $60 \%$ of the signal comes from the top Co-Pt multilayers. Assuming that the ratio of the absorption in both layers is equal to the ratio of the Kerr rotation, an assumption justified by calculations (see Supplementary Information, Part III), and that both layers are independent (that is, no spin transfer), the contributions to the Kerr signal from the top and bottom multilayers can be calculated: $\theta_{\text {top }}-\Delta \theta_{\text {top }}=c \times 0.6(1-0.6 \delta)$ and $\theta_{\text {bottom }}-\Delta \theta_{\text {bottom }}=c \times 0.4(1-0.4 \delta)$, where $c$ is the Kerr rotation of the system and $\delta$ accounts for the real demagnetization. In the parallel configuration, the total Kerr rotation is $\theta_{\mathrm{P}}=c(1-0.52 \delta)$, and thus $(\Delta \theta / \theta)_{\mathrm{P}}=-0.52 \delta$. From the measurement, we can extract $(\Delta \theta / \theta)_{\mathrm{P}}$ and then evaluate $\delta$. Thus we can evaluate the relative change of Kerr rotation in the AP state using $(\Delta \theta / \theta)_{\mathrm{AP}}=-1.0 \delta$, which is almost twice as large as in the P state.

\section{Received 21 January 2008; accepted 29 August 2008; published 28 September 2008.}

\section{References}

1. Beaurepaire, E., Merle, J.-C., Daunois, A. \& Bigot, J.-Y. Ultrafast spin dynamics in ferromagnetic nickel. Phys. Rev. Lett. 76, 4250-4253 (1996).

2. Hohlfeld, J., Matthias, E., Knorren, R. \& Bennemann, K. H. Nonequilibrium magnetization dynamics of nickel. Phys. Rev. Lett. 78, 4861-4864 (1997).

3. Scholl, A., Baumgarten, L., Jacquemin, R. \& Eberhardt, W. Ultrafast spin dynamics of ferromagnetic thin films observed by fs spin-resolved two-photon photoemission. Phys. Rev. Lett. 79 5146-5149 (1997).

4. Koopmans, B., van Kampen, M., Kohlhepp, J. T. \& de Jonge, W. J. M. Ultrafast magneto-optics in nickel: Magnetism or optics? Phys. Rev. Lett. 85, 844-847 (2000).

5. Regensburger, H., Vollmer, R. \& Kirschner, J. Time-resolved magnetization-induced second-harmonic generation from the Ni(110) surface. Phys. Rev. B 61, 14716-14722 (2000).

6. Guidoni, L., Beaurepaire, E. \& Bigot, J.-Y. Magneto-optics in the ultrafast regime: Thermalization of spin populations in ferromagnetic films. Phys. Rev. Lett. 89, 017401 (2002).

7. Koopmans, B. Handbook of Magnetism and Advanced Magnetic Materials Vol. 3, 1589-1613 (Wiley, 2007).

8. Koopmans, B., Ruigrok, J. J. M., Dalla Longa, F. \& de Jonge, W. J. M. Unifying ultrafast magnetization dynamics. Phys. Rev. Lett. 95, 267207 (2005).

9. Stam, $\mathrm{C}$ et al. Femtosecond modification of electron localization and transfer of angular momentum in nickel. Nature Mater. 6, 740-743 (2007)

10. Dalla Longa, F., Kohlhepp, J. T., de Jonge, W. J. M. \& Koopmans, B. The influence of photon angular momentum on ultrafast demagnetization in nickel. Phys. Rev. B 75, 224431 (2007).

11. Stiles, M. D. \& Miltat, J. Spin Dynamics in Confined Magnetic Structures III: Topics in Applied Physics Vol. 101, 225-308 (Springer, 2006).

12. Bedau, D. et al. Detection of current-induced resonance of geometrically confined domain walls. Phys. Rev. Lett. 99, 146601 (2007).

13. Hayashi, M., Thomas, L., Rettner, C., Moriyam, R. \& Parkin, S. S. P. Direct observation of the coherent precession of magnetic domain walls propagating along permalloy nanowires. Nature Phys. 3, 21-25 (2007).

14. Tserkovnyak, Y., Brataas, A. \& Bauer, G. E. W. Enhanced Gilbert damping in thin ferromagnetic films Phys. Rev. Lett. 88, 117601 (2002).

15. Šimánek, E. \& Heinrich, B. Gilbert damping in magnetic multilayers. Phys. Rev. B 67, 144418 (2003).

16. Costache, M. V., Sladkov, M., Watts, S. M., van der Wal, C.H \& van Wees, B. J. Electrical detection of spin pumping due to the precessing magnetization of a single ferromagnet. Phys. Rev. Lett. 97, 216603 (2006).

17. Mizukami, S., Ando, Y, Miyazaki, T. \& van Wees, B. J. Ferromagnetic resonance linewidth for $\mathrm{NM} / 80 \mathrm{NiFe} / \mathrm{NM}$ films (NM = Cu, Ta, Pd and Pt). J. Magn. Magn. Mater. 226-230, 1640 (2001).

18. Urban, R., Woltersdorf, G. \& Heinrich, B. Gilbert damping in single and multilayer ultrathin films: Role of interfaces in nonlocal spin dynamics. Phys. Rev. Lett. 87, 217204 (2001).

19. Woltersdorf, G., Mosendz, O., Heinrich, B. \& Back, C. H. Magnetization dynamics due to pure spin currents in magnetic double layers. Phys. Rev. Lett. 99, 246603 (2007).

20. Hellwig, O., Kirk, T. L., Kortright, J. B., Berger, A. \& Fullerton, E. A new phase diagram for layered antiferromagnetic films. Nature Mater. 2, 112-116 (2003).

21. Hellwig, O., Berger, A. \& Fullerton, E. Domain walls in antiferromagnetically coupled multilayer films. Phys. Rev. Lett. 91, 197203 (2003).

22. Liu, Z. Y. \& Adenwalla, S. Oscillatory interlayer exchange coupling and its temperature dependence in $[\mathrm{Pt} / \mathrm{Co}]_{3} / \mathrm{NiO} /[\mathrm{Pt} / \mathrm{Co}]_{3}$ multilayers with perpendicular anisotropy. Phys. Rev. Lett. 91, 037207 (2003).

23. Zhuravlev, M. Ye., Tsymbal, E. Y. \& Jaswal, S. S. Exchange model for oscillatory interlayer coupling and induced unidirectional anisotropy in $[\mathrm{Pt} / \mathrm{Co}]_{3} / \mathrm{NiO} /[\mathrm{Pt} / \mathrm{Co}]_{3}$ multilayers. Phys. Rev. Lett. 92 219703 (2004).

24. van Kampen, M., Kohlhepp, J. T., de Jonge, W. J. M., Koopmans, B. \& Coehoorn, R. Sub-picosecond electron and phonon dynamics in nickel. J. Phys. Condens. Matter 17, 6823-6834 (2005).

25. Satoshi, K., Yasuo, A., Terunobu, M. \& Shigemi, M. Temperature dependencies of spin-diffusion lengths of Cu and Ru layers. Jpn. J. Appl. Phys. 45, 3892-3895 (2006).

26. Worledge, D. C. \& Trouilloud, P. L. Magnetoresistance measurement of unpatterned magnetic tunnel junction wafers by current-in-plane tunnelling. Appl. Phys. Lett. 83, 84-86 (2003).

Supplementary Information accompanies the paper at www.nature.com/naturephysics.

\section{Acknowledgements}

This research was supported by NanoNed, a nanotechnology programme of the Dutch Ministry of Economic Affairs.

\section{Author information}

Reprints and permissions information is available online at http://npg.nature.com/reprintsandpermissions. Correspondence and requests for materials should be addressed to G.M. 